\title{
Pex4Fun: A Web-Based Environment for Educational Gaming via Automated Test Generation
}

\author{
Nikolai Tillmann, Jonathan de Halleux \\ Microsoft Research \\ One Microsoft Way \\ Redmond, WA, USA \\ Email: \{nikolait,jhalleux $@$ microsoft.com
}

\author{
Tao Xie \\ Department of Computer Science \\ University of Illinois at Urbana-Champaign \\ Urbana, IL, USA \\ Email: taoxie@illinois.edu
}

\author{
Judith Bishop \\ Microsoft Research \\ One Microsoft Way \\ Redmond, WA, USA \\ Email: jbishop@microsoft.com
}

\begin{abstract}
Pex4Fun (http://www.pex4fun.com/) is a web-based educational gaming environment for teaching and learning programming and software engineering. Pex4Fun can be used to teach and learn programming and software engineering at many levels, from high school all the way through graduate courses. With Pex4Fun, a student edits code in any browser - with Intellisense - and Pex4Fun executes it and analyzes it in the cloud Pex4Fun connects teachers, curriculum authors, and students in a unique social experience, tracking and streaming progress updates in real time. In particular, Pex4Fun finds interesting and unexpected input values (with Pex, an advanced test-generation tool) that help students understand what their code is actually doing. The real fun starts with coding duels where a student writes code to implement a teacher's secret specification (in the form of sample-solution code not visible to the student). Pex4Fun finds any discrepancies in behavior between the student's code and the secret specification. Such discrepancies are given as feedback to the student to guide how to fix the student's code to match the behavior of the secret specification.

This tool demonstration shows how Pex4Fun can be used in teaching and learning, such as solving coding duels, exploring course materials in feature courses, creating and teaching a course, creating and publishing coding duels, and learning advanced topics behind Pex4Fun.
\end{abstract}

\section{INTRODUCTION}

Teaching and learning programming and software engineering have received a lot of attention from researchers and educators. Various programming environments such as Alice [13], Scratch [12], [11], and Greenfoot [9], have been provided for instilling fun into students' programming-learning experiences, especially for beginner learners. These programming environments have achieved significant success [22], [6] in helping teach and learn programming concepts for beginner learners. However, these environments typically target at some specialized programming languages other than mainstream programming languages. In addition, these environments primarily target at teaching and learning programming without focusing on software engineering.

As part of our research efforts on educational software engineering [24] and browser-based software for technology transfer [3], we have developed a web-based educational gaming environment for teaching and learning programming and software engineering, called Pex4Fun ${ }^{1}$ [19] (denoting Pex

\footnotetext{
${ }^{1}$ http://www.pex4fun.com/
}

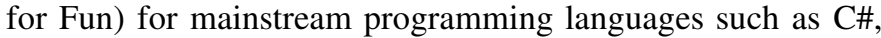
Visual Basic, and F\#. It works on any web-enabled device, even a smart phone [20]. It comes with an auto-completing code editor, providing a user with instant feedback similar to the code editor in Microsoft Visual Studio. It is a cloud application with the data in the cloud, enabling a user to use it anywhere where Internet connection is available.

New learners of programming can play games there to master basic programming concepts. Learners of software engineering can play games there to master advanced programming concepts and software engineering concepts. Even experienced software engineers can play games to improve their skills while having fun.

Teachers can create virtual classrooms in the form of courses by customizing existing learning materials and games or creating new materials and games; teachers can enjoy the benefits of automated grading of game exercises assigned to students.

Behind the scene of Pex4Fun, its underlying technology is called dynamic symbolic execution [7], [14], [4], which has been realized by a white-box testing tool called Pex [17], the backbone of Pex4Fun. Pex4Fun has been gaining popularity in the community: since it was released to the public in June 2010, the number of clicks of the "Ask Pex!" button (indicating the attempts made by users to solve games at Pex4Fun) has reached over 1.3 millions $(1,318,839)$ as of August 29, 2013.

The rest of the paper is organized as follows. Section II presents the background information on the technology and supporting tool underlying the Pex4Fun environment. Section III presents the overview of the Pex4Fun environment. Section IV presents the tool usage scenarios. Section V presents related work. Section VI discusses the potential impact of the environment to the broader community.

\section{Background: Automated Test Generation}

We next present the underlying technology (dynamic symbolic execution) and supporting tool (Pex) for the Pex4Fun environment.

Dynamic symbolic execution (DSE) [7], [14], [4] is a variation of symbolic execution [8], [5] and leverages runtime information from concrete executions. DSE is often conducted in iterations to systematically increase code coverage such as 


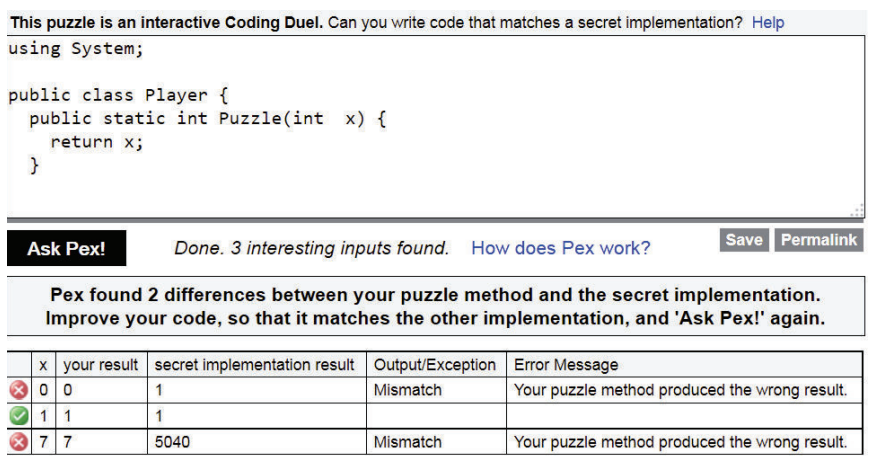

Fig. 1. The user interface of the Pex4Fun website

block or branch coverage. In each iteration, DSE executes the program under test with a test input, which could be a default or randomly generated input in the first iteration or an input generated in one of the previous iterations. During the execution of the program under test, DSE performs symbolic execution in parallel to collect symbolic constraints on program inputs obtained from predicates in branch statements along the execution. The conjunction of all symbolic constraints along an executed path is called the path condition. Then DSE flips a branching node in the executed path to construct a new path that shares the prefix to the node with the executed path, but then deviates and takes a different branch. DSE relies on a constraint solver to (1) check whether such a flipped path is feasible; if so, (2) compute a satisfying assignment — such assignment forms a new test input whose execution will follow along the flipped path.

Pex [17] is an automatic white-box test-generation tool for .NET, based on DSE. Pex has been integrated into Microsoft Visual Studio as an add-in. Pex can generate test inputs that can be integrated with various unit testing frameworks such as NUnit $^{2}$ and MSTest ${ }^{3}$. Pex was applied to test a core component of the .NET architecture, which had already been extensively tested over five years by approximately 40 testers within $\mathrm{Mi}$ crosoft. The component is the basis for other libraries, which are used by thousands of developers and millions of end users. Pex found various issues in this core component, including a serious issue. Pex was used in classroom teaching at different universities as well as various tutorials both within Microsoft (such as internal training of Microsoft developers) and outside Microsoft (such as tutorials at .NET user groups) [23].

\section{Overview OF PEX4Fun}

The Pex4Fun environment [19] includes coding duels as the major type of games for learning various concepts and skills in programming and software engineering. Figure 1 shows a screen snapshot of the user interface of the Pex4Fun website, which shows an example coding duel being solved by a student. Figure 2 shows the workflow of creating and playing the example coding duel.

In particular, in a coding duel, a student's task is to implement the Puzzle method (shown on the top-right side

\footnotetext{
${ }^{2} \mathrm{http}: / /$ www.nunit.org/

${ }^{3} \mathrm{http}: / / \mathrm{msdn}$.microsoft.com/en-us/library/ms182489(v=vs.80).aspx
}

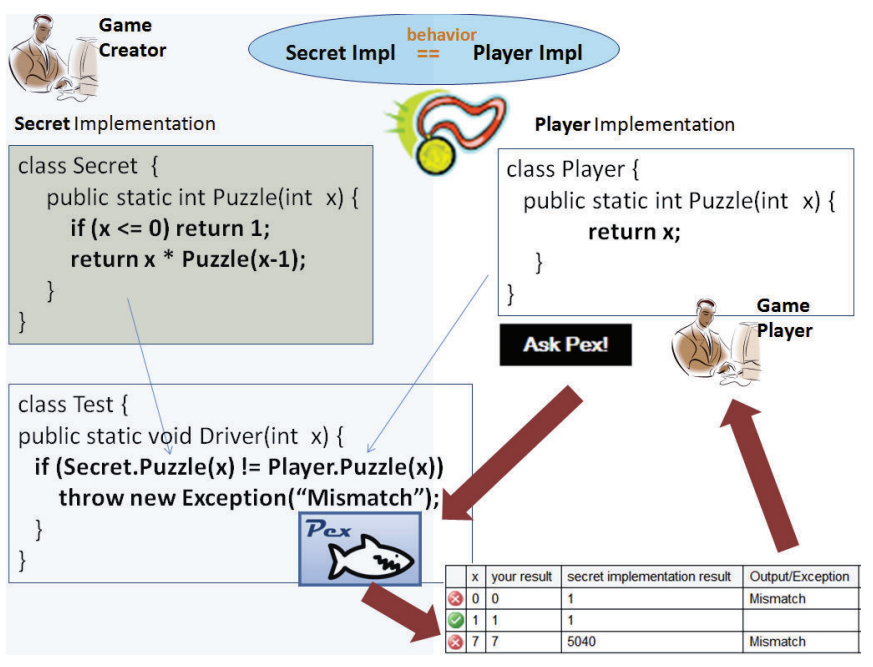

Fig. 2. The workflow of creating and playing a coding duel

of Figure 2 and in Figure 1) to have exactly the same behavior as another secret Puzzle method, which is never visible to the student (shown on the top-left side of Figure 2), based on feedback in the form of some selected values where the student's current version of the Puzzle method behaves differently as well as some selected values where it behaves the same way (shown near the bottom of Figure 1 and near the right-bottom of Figure 2).

In the example coding duel, the Puzzle method is public static int Puzzle(int $x$ ). The feedback given to the student on some selected input values is displayed as a table near the bottom of the screen (in Figure 1). A table row beginning with a check mark in a green circle indicates that the corresponding test is a passing test. Formally, the return values of the secret implementation and student implementation (i.e., the Puzzle method implementation) are the same for the same test input (i.e., the Puzzle method argument value). A table row started with a red circle with a cross indicates that the corresponding test is a failing test: the return values of the secret implementation and student implementation are different for the same test input. In the table, the second column " $x$ " indicates the test input. The third and fourth columns "your result" and "secret implementation result" indicate the return values of the student implementation and secret implementation, respectively. The last two columns "Output/Exception" and "Error Message" give more details for the failing tests.

A student can solve a simple coding duel with iterations each with the following five main steps. (1) Click an example coding duel from the Pex4Fun website; then the student can see a student implementation that does not do much. (2) Click "Ask Pex!" to see how the student implementation differs from the secret implementation [16]. (3) Compare the student implementation's result to the secret implementation's result. (4) Analyze the differences and change the code to match the secret implementation's results for all input values or as many input values as the student can. (5) Click "Ask Pex!" again. Repeat this process until the student wins the coding duel (i.e., 
no failing tests being reported in the table by Pex) or cannot make any progress.

\section{TOOL USAGE ScEnarios}

Pex4Fun (http://www.pex4fun.com/) brings programming and software engineering with fun to a student's web browser. Enjoying fun experiences, a student can write, compile, and run code in order to learn programming concepts, practice programming and software-engineering skills, and analyze the behavior of code interactively. In particular, this tool demonstration shows how Pex4Fun can be used to teach and learn programming and software engineering via the following aspects.

- Solve puzzles. The main Puzzle method used in Pex4Fun can take parameters and return values. In order to run such a puzzle method, someone must provide argument values. A student can click “Ask Pex!”, and then Pex [17], the underlying test-generation engine, automatically finds interesting argument values by analyzing the code.

- Solve coding duels. A coding duel is an interactive puzzle. In a coding duel, a student's task is to implement the Puzzle method to have exactly the same behavior as another secret Puzzle method. To start with a simple coding duel, the student can click an example coding duel from the website.

- Explore course materials in feature courses. The current feature courses include C\# for fun (for C\# learners), Parameterized Unit Testing [21], [18] (for developertesting learners [25], [23]), Code Contracts [1] in .NET (for specification learners).

- Create and teach a course. Pex4Fun can be used to build interesting, engaging, demanding classes on mathematics, algorithms, programming languages, software engineering, or problem solving in general. Teachers can use an integrated wiki to author classes built upon puzzles and coding duels. In particular, a teacher combines existing pages into a course. The pages might have been written by the teacher or by any other author. The teacher invites students to the course by sharing a registration link with them. A course can have multiple teachers. Any user can become a student by registering for a course through the registration link. The student can then work through the pages that are part of the course.

- Create and publish coding duels. A user can create and publish coding duels via five main steps. (1) Sign in, so that Pex4Fun can maintain coding duels for the user. (2) Write a specification starting from a puzzle template where the user can write the specification as a Puzzle method that takes inputs and produces an output. (3) Create the coding duel by clicking a button "Turn This Puzzle Into A Coding Duel" (appearing after the user clicks “Ask Pex!”). (4) Edit visible program text next by clicking the coding duel Permalink URL, which opens the coding duel, and by filling in a slightly more useful outline of the implementation (with optional comments) that somebody else will eventually complete. (3) Publish after the user finishes editing the visible Puzzle method text by clicking the "Publish" button.

\section{RELATED WORK}

Previous work [10], [2] advocated using a "Game First" approach to teaching introductory programming. The compelling level of course assignments and example contents has been much emphasized. With compelling assignments, students are much more likely to learn because they are interested, and visual components in developed games allow students to more easily see mistakes in their code. The Pex4Fun environment shares a similar motivation but putting the realization of the compelling factor in the style of interactive-gaming-based coding duels.

Maloney et al. [12] designed a visual game around the programming concepts of loops and arrays in introductory courses. In particular, students could change loops and arrays in programs in an interactive and visual way. Then the game provides immediate feedback and helps students visualize program executions. Their evaluation results show that the game helped students better understand these programming concepts. The Pex4Fun environment also provides immediate feedback to students in terms of their student implementation's behaviors compared against the secret implementation.

Spacco et al. [15] developed Marmoset ${ }^{4}$, an automated snapshot, submission and testing system. The system captures snapshots of students' programming projects to a centralized code repository whenever the students save their source files. Such a collected fine-grained revision history offers teachers a unique perspective into the development process for students. Similar to this aspect, the Pex4Fun environment also captures snapshots of students' revisions of the student implementation whenever the students click the "Ask Pex!" button to request feedback from Pex. Similarly, teachers can investigate the duelsolving process for students. When using Marmoset, students can also explicitly submit projects to the Marmoset system to request Marmoset to run these submissions against a suite of unit tests developed by the teachers to evaluate the functional correctness of a submission. In contrast, the teachers are not required to develop a suite of tests for evaluating functional correctness of a student implementation against the secret implementation. Instead, Pex is used to serve this evaluation purpose. In addition, tests generated by Pex are not a fixed set, unlike the tests used by Marmoset: Pex generates different tests depending on the program behavior of a student's submitted student implementation, accomplishing the goal of personalized or customized feedback for each student.

\section{Potential Impact to Broader Community}

The Pex4Fun environment has been gaining popularity in the community: since it was released to the public in June 2010, the number of clicks of the "Ask Pex!" button (indicating the attempts made by users to solve games at Pex4Fun) has reached over 1.3 millions $(1,318,839)$ as of August 29,

\footnotetext{
${ }^{4}$ http://marmoset.cs.umd.edu/
} 
2013. Pex4Fun has provided a number of open virtual courses including learning materials along with games used to reinforce students' learning (http://www.pex4fun.com/Page.aspx\# learn/courses). Pex4Fun was adopted as a major platform for assignments in a graduate software engineering course. A coding-duel contest (http://www.pex4fun.com/icse2011) was held at a major software engineering conference (ICSE 2011) for engaging conference attendees to solve coding duels in a dynamic social contest.

The Pex4Fun environment serves as a high-impact example to show that a sophisticated software engineering technique (e.g., automated test generation) can be successfully leveraged to underpin educational gaming and automatic grading in a web-based system that can scale to hundreds of thousands of users.

\section{ACKNOWLEDGMENT}

Tao Xie's work is supported in part by NSF grants CCF-0845272, CCF-0915400, CNS-0958235, CNS-1160603, a NIST grant, a Microsoft Research Software Engineering Innovation Foundation Award, and NSF of China No. 61228203.

\section{REFERENCES}

[1] M. Barnett, M. Fähndrich, P. de Halleux, F. Logozzo, and N. Tillmann. Exploiting the synergy between automated-test-generation and programming-by-contract. In Proceedings of the 31st International Conference on Software Engineering (ICSE), Companion, pages 401402, 2009.

[2] J. D. Bayliss. Using games in introductory courses: tips from the trenches. In Proceedings of the 40th ACM Technical Symposium on Computer Science Education (SIGCSE 2009), pages 337-341, 2009.

[3] J. Bishop, J. de Halleux, N. Tillmann, N. Horspool, D. Syme, and T. Xie. Browser-based software for technology transfer. In Proceedings of the 2011 Annual Research Conference of the South African Institute for Computer Scientists and Information Technologists (SAICSIT 2011), Industry Oriented Paper, pages 338-340, 2011.

[4] C. Cadar and D. R. Engler. Execution generated test cases: How to make systems code crash itself. In Proceedings of the 12th International SPIN Workshop on Model Checking Software (SPIN 2005), pages 2-23, 2005.

[5] L. A. Clarke. A system to generate test data and symbolically execute programs. IEEE Trans. Softw. Eng., 2(3):215-222, 1976.

[6] S. Fincher, S. Cooper, M. Kölling, and J. Maloney. Comparing Alice, Greenfoot \& Scratch. In Proceedings of the 41st ACM Technical Symposium on Computer Science Education (SIGCSE 2010), pages 192 193, 2010.

[7] P. Godefroid, N. Klarlund, and K. Sen. DART: directed automated random testing. In Proceedings of the 2005 ACM SIGPLAN Conference on Programming Language Design and Implementation (PLDI 2005), pages 213-223, 2005

[8] J. C. King. Symbolic execution and program testing. Commun. ACM, 19(7):385-394, 1976

[9] M. Kölling and P. Henriksen. Game programming in introductory courses with direct state manipulation. In Proceedings of the 10th Annual SIGCSE Conference on Innovation and Technology in Computer Science Education (ITiCSE 2005), pages 59-63, 2005.

[10] S. Leutenegger and J. Edgington. A games first approach to teaching introductory programming. In Proceedings of the 38th SIGCSE Technical Symposium on Computer Science Education (SIGCSE 2007), pages 115-118, 2007.
[11] D. J. Malan and H. H. Leitner. Scratch for budding computer scientists. In Proceedings of the 38th SIGCSE Technical Symposium on Computer Science Education (SIGCSE 2007), pages 223-227, 2007.

[12] J. H. Maloney, K. Peppler, Y. Kafai, M. Resnick, and N. Rusk. Programming by choice: urban youth learning programming with Scratch. In Proceedings of the 39th SIGCSE Technical Symposium on Computer Science Education (SIGCSE 2008), pages 367-371, 2008.

[13] R. Pausch, T. Burnette, A. Capeheart, M. Conway, D. Cosgrove, R. DeLine, J. Durbin, R. Gossweiler, S. Koga, and J. White. A brief architectural overview of Alice, a rapid prototyping system for virtual reality. IEEE Computer Graphics and Applications, pages 195-203, May 1995.

[14] K. Sen, D. Marinov, and G. Agha. CUTE: a concolic unit testing engine for C. In Proceedings of the 10th European Software Engineering Conference held jointly with 13th ACM SIGSOFT International Symposium on Foundations of Software Engineering (ESEC/FSE 2005), pages 263272,2005

[15] J. Spacco, D. Hovemeyer, W. Pugh, J. Hollingsworth, N. Padua-Perez, and F. Emad. Experiences with Marmoset: Designing and using an advanced submission and testing system for programming courses. In Proceedings of the 11th Annual Conference on Innovation and Technology in Computer Science Education (ITiCSE 2006), pages 13-17, 2006.

[16] K. Taneja and T. Xie. DiffGen: Automated regression unit-test generation. In Proceedings of the 23rd IEEE/ACM International Conference on Automated Software Engineering (ASE 2008), pages 407-410, 2008.

[17] N. Tillmann and J. de Halleux. Pex - white box test generation for .NET. In Proceedings of the 2nd International Conference on Tests And Proofs (TAP 2008), pages 134-153, 2008.

[18] N. Tillmann, P. de Halleux, and T. Xie. Parameterized unit testing: Theory and practice. In Proceedings of the 32nd International Conference on Software Engineering (ICSE 2010), Companion Volume, Tutorial, pages 483-484, 2010

[19] N. Tillmann, J. D. Halleux, T. Xie, S. Gulwani, and J. Bishop. Teaching and learning programming and software engineering via interactive gaming. In Proceedings of the 35th International Conference on Software Engineering (ICSE 2013), Software Engineering Education (SEE), pages 1117-1126, 2013

[20] N. Tillmann, M. Moskal, J. de Halleux, M. Fahndrich, J. Bishop, A. Samuel, and T. Xie. The future of teaching programming is on mobile devices. In Proceedings of the 17th Annual Conference on Innovation and Technology in Computer Science Education (ITiCSE 2012), pages 156-161, 2012.

[21] N. Tillmann and W. Schulte. Parameterized unit tests. In Proceedings of the 10th European Software Engineering Conference held jointly with 13th ACM SIGSOFT International Symposium on Foundations of Software Engineering (ESEC/FSE 2005), pages 253-262, 2005.

[22] I. Utting, S. Cooper, M. Kölling, J. Maloney, and M. Resnick. Alice, Greenfoot, and Scratch - a discussion. Trans. Comput. Educ., 10:17:117:11, November 2010.

[23] T. Xie, J. de Halleux, N. Tillmann, and W. Schulte. Teaching and training developer-testing techniques and tool support. In Proceedings of the 25th Annual ACM Conference on Systems, Programming, Languages, and Applications: Software for Humanity (SPLASH 2010), Educators' and Trainers' Symposium, pages 175-182, 2010.

[24] T. Xie, N. Tillmann, and J. de Halleux. Educational software engineering: Where software engineering, education, and gaming meet. In Proceedings of the 3rd International Workshop on Games and Software Engineering (GAS 2013), pages 36-39, 2013.

[25] T. Xie, N. Tillmann, J. de Halleux, and W. Schulte. Future of developer testing: Building quality in code. In Proceedings of the FSE/SDP Workshop on the Future of Software Engineering Research (FoSER 2010), pages 415-420, 2010. 